\title{
UAVSAR: A New NASA Airborne SAR System for Science and Technology Research
}

\author{
Paul A. Rosen, Scott Hensley, Kevin Wheeler, Greg Sadowy, Tim Miller, Scott Shaffer, Ron Muellerschoen, Cathleen \\ Jones, Howard Zebker†, Søren Madsen \\ Radar Science and Engineering Section, Jet Propulsion Laboratory, California Institute of Technology, Pasadena, CA/USA, \\ Paul.A.Rosen@jpl.nasa.gov \\ $†$ Stanford University, Palo Alto, CA, 94305
}

\begin{abstract}
NASA's Jet Propulsion Laboratory is currently building a reconfigurable, polarimetric L-band synthetic aperture radar (SAR), specifically designed to acquire airborne repeat track SAR data for differential interferometric measurements. Differential interferometry can provide key deformation measurements, important for studies of earthquakes, volcanoes and other dynamically changing phenomena. Using precision real-time GPS and a sensor controlled flight management system, the system will be able to fly predefined paths with great precision. The expected performance of the flight control system will constrain the flight path to be within a $10 \mathrm{~m}$ diameter tube about the desired flight track. The radar will be designed to be operable on a UAV (Unpiloted Arial Vehicle) but will initially be demonstrated on a on a NASA Gulfstream III. The radar will be fully polarimetric, with a range bandwidth of $80 \mathrm{MHz}$ ( $2 \mathrm{~m}$ range resolution), and will support a $16 \mathrm{~km}$ range swath. The antenna will be electronically steered along track to assure that the antenna beam can be directed independently, regardless of the wind direction and speed. Other features supported by the antenna include elevation monopulse and pulse-to-pulse re-steering capabilities that will enable some novel modes of operation. The system will nominally operate at $45,000 \mathrm{ft}(13800 \mathrm{~m})$. The program began as an Instrument Incubator Project (IIP) funded by NASA Earth Science and Technology Office (ESTO).
\end{abstract}

Index Terms - electronically scanned array, interferometry, radar, SAR, UAV.

\section{INTRODUCTION}

The solid earth science community is seeking earth deformation measurements at a variety of temporal scales, from seconds to decades. The NASA Solid Earth Science Working Group has recommended an observational program that includes both airborne and spaceborne capabilities and this is reflected in the NASA Earth Science Enterprise strategic plan [1]. Ultimately, scientists would like to have Earth deformation measurements on an hourly basis with global access, objectives best supported by a spaceborne highorbit (e.g. geosynchronous) constellation of repeat-pass interferometric SAR satellites [2,3]. The recommended first step in this observational program is a low-earth-orbit deformation satellite with a repeat period of roughly one week. The sub-orbital radar program enters NASA's Earth Science program as a key supplemental capability, providing repeat-pass measurements at time scales much smaller than one week, potentially as short as twenty minutes.
Understanding the time varying nature of rapidly deforming features such as some volcanoes and glaciers or deformation from post seismic transients requires observational sampling intervals of a day or less to capture and model such events.

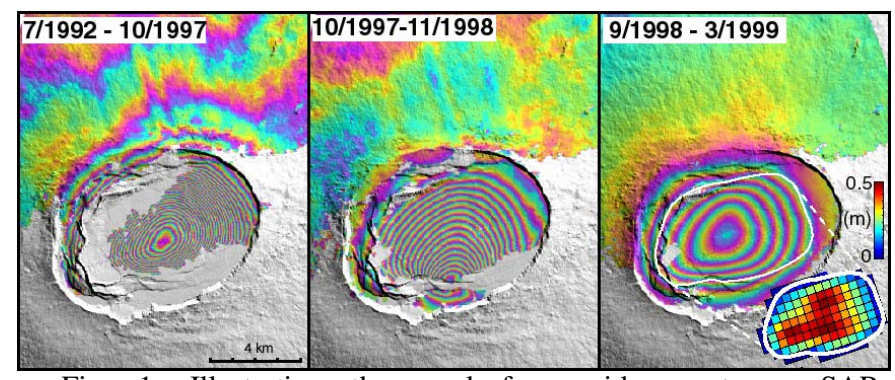

Fig. 1: Illustrating the need for rapid repeat-pass SAR measurements. The three interferograms shown in this figure are from a set of 5 C-band ERS images acquired over a volcanic caldera in the Galapagos Islands. Color indicates the amount of deformation (color bar at right) in each interferogram between the times specified in upper left of each panel. Clearly, a substantial amount of deformation is occurring in this caldera over extended time periods, and this particular signal is highly undersampled in time, a problem UAVSAR is designed to address.

Figure 1 provides one example of the need for rapid sampling of a dynamic event. In this case, a volcanic caldera on the Galapagos Islands is seen to experience meter scale deformation in a highly unpredictable manner over a period of years. Because only few images have been acquired over a span of seven years, it is impossible to determine if the deformation is periodic, episodic, fast or slow, and therefore the mechanisms of magma transport, chamber pressurization, and potential release cannot be modeled in anything more than a cursory way. A rapid-repeat, yet persistent interferometric system will provide the needed measurements for this kind of dynamic situation. Scientific disciplines where regular and repeated interferometric measurements at fine spatial resolution are needed include:

- The study of crustal faults and the earthquake cycle, through preseismic, co-seismic, and perhaps most importantly, post-seismic deformation.

- The study of volcano dynamics and hazards through mapping of the temporal history of the eruption cycle, including small scale deformation leading to an eruption 
and much larger scale deformation measurements during an eruption.

- The study of ice dynamics and its relation to mass balance and sea level rise, including surges and pulses of glaciers and ice sheets, the effects of tidal oscillations on glacier and ice sheet stability, and the nature and implications of calving dynamics and ice sheet disintegration.

- The study of local sea ice dynamics, measuring strain rates of deforming ice and the nature of fracturing at fine resolution.

- The study of time-varying evaporation and hydraulic properties of soils as important inputs to the surface water and energy cycle.

- The study of above ground biomass extent, distribution, stress due to environmental change, and relation to forest structure.

In addition to providing unprecedented temporal detail of deformation of dynamic processes, the suborbital radar will be a test bed for understanding the operational requirements of a science instrument optimized for rapid repeat observations. For example, the rate of random surface change of different surface types over different time scales, resolution scales, and weather conditions is not well known. This temporal change component can greatly influence the amount of data needed and the way in which an experiment would be designed. This is a capability that the currently operational NASA AIRSAR system has demonstrated but cannot practically support for science experiments in its current configuration due to lack of track repeatability and beam pointing limitations.

Although satellites have been used for repeat track interferometric (RTI) SAR mapping for close to 20 years, repeat track interferometry is much more difficult to implement from an airborne platform. Several organizations have acquired experimental airborne RTI data, however, without developing a capability to acquire significant amounts of high quality RTI data. The primary reason for this state of affairs is that 1) It is difficult to fly the same or nearly the same pass twice in the air, due to wind gusts, turbulence, etc. and 2) it is difficult to maintain the same antenna pointing on repeated passes due to varying cross-winds that lead to varying yaw angles.

The UAVSAR project can also serve as a technology test bed. As a modular instrument with numerous plug-and-play components, it will be possible to test new technologies for airborne and spaceborne applications with relatively little difficulty. Joint experiments with other radar instruments, for example radars flying in formation with an active link between platforms, can also be accommodated without redesign of the system.
The project started as a proposal submitted to the NASA 2002 Instrument Incubator Program (IIP) to develop a repeat track measurement capability as an augmentation to the existing AIRSAR system. NASA accepted the proposal but directed that the proposed capability be fielded on a UAV or MPV platform to support the long-term interests of the science community. After a year of study and experiments, NASA directed JPL to proceed with a full-scale implementation. This paper outlines the status of the project, including the highlevel radar design.

\section{SYSTEM REQUIREMENTS OVERVIEW}

Repeat track interferometry not only requires that the phase centers of the radar antenna locations for the individual tracks are approximately coincident, it is also essential that the antenna look directions are identical to within a fraction of the beamwidth. Given that the wind can be substantially different at different times, even if the platform is capable of accurately repeating the desired track, the yaw angle of the aircraft can vary widely on different tracks due to different wind condition aloft. This we intend to mitigate by electronically steering a flush mounted antenna to the desired direction. As such, the UAVSAR system is a tight blending of platform and sensor capabilities.

Given that the temporal separation of the acquired track will have a wide range (minutes to years), it is required that the radar wavelength be long, however, at the same time the largest possible bandwidth is desired to increase the so-called critical baseline (maximal separation between antenna phase centers supporting interferometric observations). L-band is found to be a very attractive compromise with a quarter meter wavelength and $80 \mathrm{MHz}$ of available bandwidth. Given an assumed operating altitude of $45,000 \mathrm{ft}(13800 \mathrm{~m})$, a near angle of incidence of approximately $25^{\circ}$, and an $80 \mathrm{MHz}$ signal bandwidth - equivalent to a 2 meter slant range resolution, the perpendicular critical baseline is

$$
B_{c \perp}=\frac{\rho \lambda \tan \theta}{2 \Delta \rho} \approx \frac{15,800 m 0.24 m 0.6}{2 \cdot 2 m} \approx 600 m
$$

Although repeat track interferometry will generally work well over flat terrain, even at track separations up to on the order of a 1/10th of the critical baseline, practical effects such as terrain relief, knowledge of local elevations, slopes, and volume scattering will mean that robust RTI performance requires baselines significantly shorter. Based on our analysis, a 10 meter baseline will be acceptable in most situations, however, repeat track deviations as small as 1 meter would be very desirable.

The key radar instrument capability that supports robust repeat-pass deformation measurements is electronic steering 
of the antenna. The L-band antenna is quite large to place on a mechanical gimbal, and doing so would tie the radar system to a particular airframe, limiting its portability to other platforms. Using an electronically steered array, with beam pointing tied to the attitude of the aircraft, the portable repeatpass radar can be realized. Such a system requires:

- Electronic steering of the antenna beam with $1^{\circ}$ accuracy over a range of $\pm 20^{\circ}$ (goal $\pm 45^{\circ}$ ) in azimuth, so that the repeat pass pointing requirements can be achieved for a wide variety of wind conditions aloft.

- Steering of the antenna must be linked to the inertial navigation unit (INU) attitude measurements with an update rate capability of less than one second.

The flight track and radar electronic pointing capability desired for airborne repeat pass observations are illustrated in Fig. 2.

\section{RADAR OVERVIEW}

The proposed radar for the UAV platform is a compact podmounted polarimetric L-band radar for repeat-pass observations, The system will demonstrate key measurements

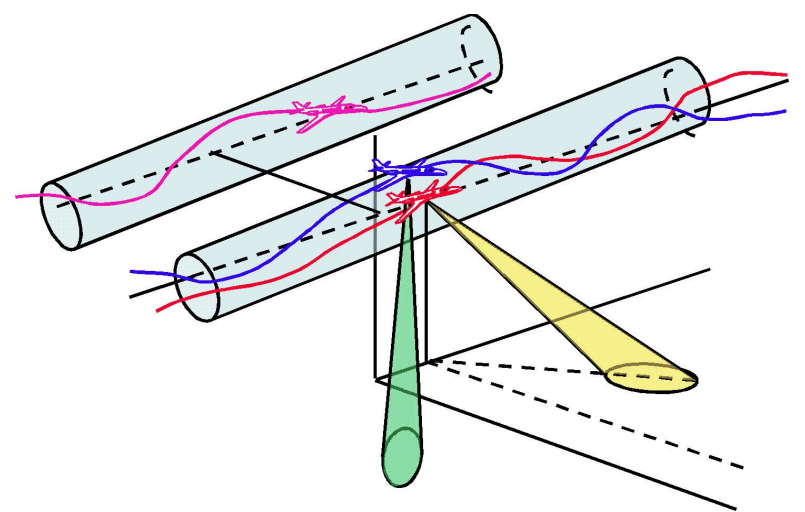

Fig. 2. To ensure high interferometric correlation for deformation measurements, the platform must repeat the trajectory within a specified tube (red and blue aircraft above). Electronic beam steering will compensate for the different aircraft yaw angles between passes. High resolution topographic mapping or tomographic imaging studies may also be supported by flying well defined baselines flying on a trajectory displaced by a spatial baseline from the reference trajectory as illustrated by the purple aircraft.

including:

- $\quad$ Precision topography change for monitoring earthquakes

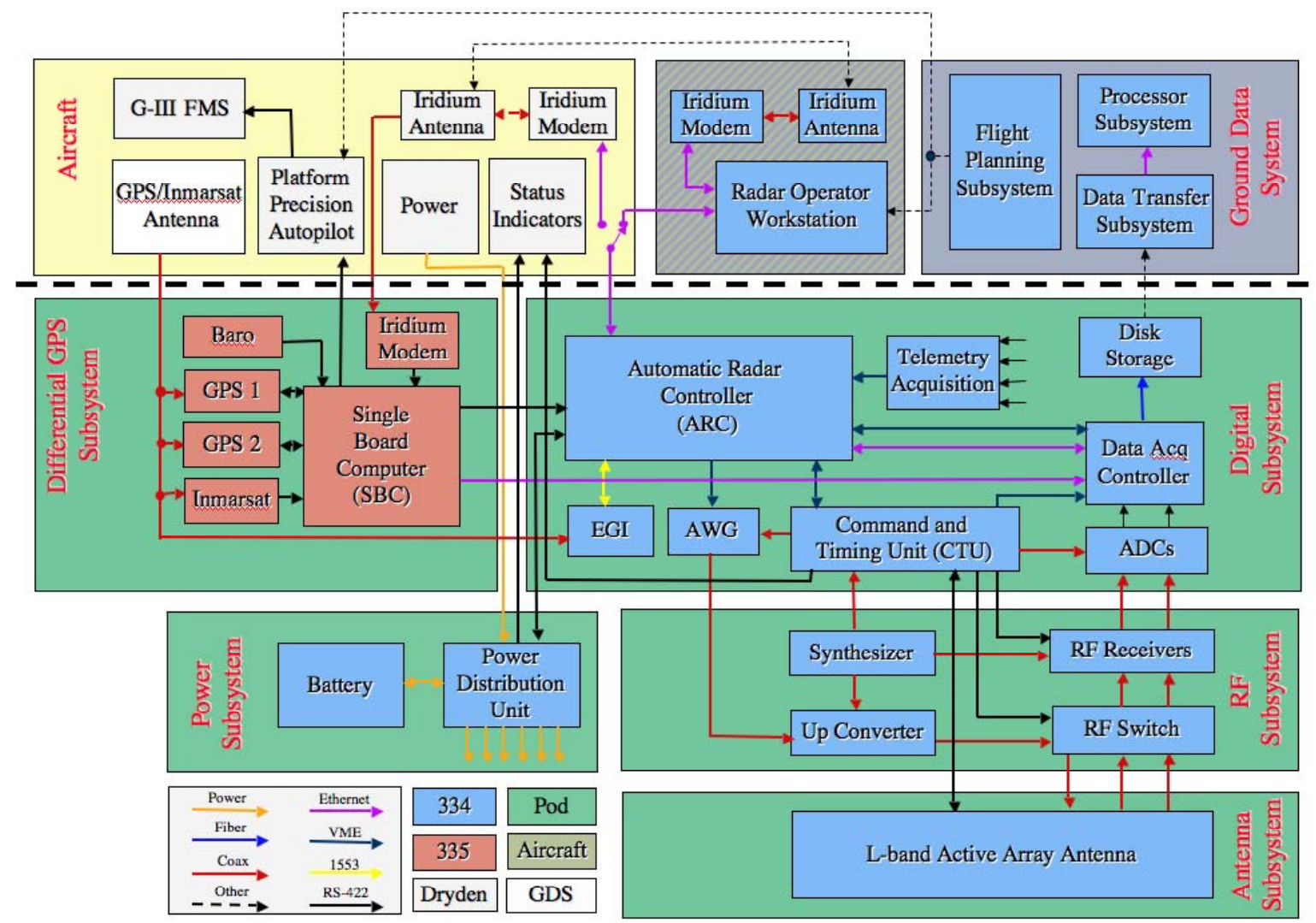

Fig. 3. UAVSAR Radar Block Diagram, including aircraft, real-time differential GPS, and ground system interfaces. Color is used to code the various signal and control paths for the radar as indicated at the bootm left of the figure. The Iridium communication system can be used to communicate with the radar and GPS systems while in flight. In particular, GPS satellite corrections can be loaded when the aircraft is out of range of the inmarsat-based correction system. 
both during and after a seismic event, for monitoring volcanic activity and for monitoring anthropogenic induced surface change such as subsidence induced by oil or water withdrawal, or other displacements of the surface from tunneling activities.

- Polarimetric interferometry, which can provide NASA with measurements of forest structure and sub-canopy topography.

- Polarimetric tomography, mapping in detail the vertical structure of a vegetated area.

Optional later additions to the system might include a second across-track antenna for single-pass interferometry (elevation mapping capability), a second antenna mounted along-track for along-track interferometry (velocity and current measurements), and additional frequencies of operation for expanded science applications

\section{A. Radar Parameters}

Based on the science objectives and UAV platform characteristics, the key parameters of the radar design include are given in Table I.

TABLE I

RADAR PARAMETERS

\begin{tabular}{|l|c|}
\hline \multicolumn{1}{|c|}{ Parameter } & Value \\
\hline Frequency & $1.26 \mathrm{GHz}(.2379 \mathrm{~m})$ \\
\hline Bandwidth & $80 \mathrm{MHz}$ \\
\hline Pulse Duration & $30 \mathrm{~ms}$ \\
\hline Polarization & $16 \mathrm{~km}$ \\
\hline Range Swath & $25^{\circ}-60^{\circ}$ \\
\hline Look Angle Range & $2.0 \mathrm{~kW}$ \\
\hline Transmit Power & $0.5 \mathrm{~m} 1.6 \mathrm{~m}$ \\
\hline Antenna Size & $2000-18000 \mathrm{~m}$ \\
\hline Altitude Range & $100-250 \mathrm{~m} / \mathrm{s}$ \\
\hline Ground Speed Range & \\
\hline
\end{tabular}

In the following sections, we will outline the radar design for the L-band polarimetric RTI radar and its expected performance. We will also describe the hardware configuration and potential opportunities for technology demonstration.

\section{B. Hardware Configuration}

The radar instrument is made up of three major subsystems: the RF electronics subsystem (RFES), the digital electronics subsystem (DES) and the antenna subsystem. Fig. 3 is a simplified instrument block diagram of the L-band radar.

The RFES performs the transmit chirp generation, frequency up-conversion, filtering, and amplification during signal transmission. The RFES also controls the routing of the radar signal and the calibration signal.

The DES performs overall control and timing for the radar, frequency down-converts and digitizes the received echo, and routes the data to on-board data storage. The dual-channel digital receiver employs two high-speed analog-to-digital converters (ADCs) capable of handling down converted Lband signals. Filtering is performed by a combination of analog and digital filters implemented on field-programmable gate arrays (FPGAs). Radar data is recorded onto a $1.2 \mathrm{~TB}$ raid array along with associated metrology and radar telemetry information. The system includes the flexibility to operate both in interferometric and multi-frequency configurations even if the timing and bandwidth between the two frequencies are asynchronous.

The antenna subsystem performs beam steering, transmission, and high power amplification on transmit and low noise amplification on receive. The antenna is a dualpolarization corporate-fed planar phased-array with $2 \times 12 \mathrm{~T} / \mathrm{R}$ modules and phase shifters for electronic beam steering from radar pulse-to-pulse. The peak transmit power for each T/R module is $100 \mathrm{~W}$ and the combined power of the $24 \mathrm{~T} / \mathrm{R}$ modules is approximately $2.0 \mathrm{~kW}$. Typical efficiency for Lband solid state amplifiers (SSPAs) is $40 \%$. On the transmit end, there will be a polarization switch to direct the transmit signal to either the $\mathrm{H}$ or $\mathrm{V}$-polarization feed of the antenna element. On the receive end, each T/R module will have two receiver front-ends (pre-select filter, high power limiter, and low-noise amplifier) to accommodate radar echoes from both the $\mathrm{H}$ and $\mathrm{V}$-polarizations. The high degree of phase fidelity required for interferometric applications has lead to the inclusion of an active calibration scheme to track the phase

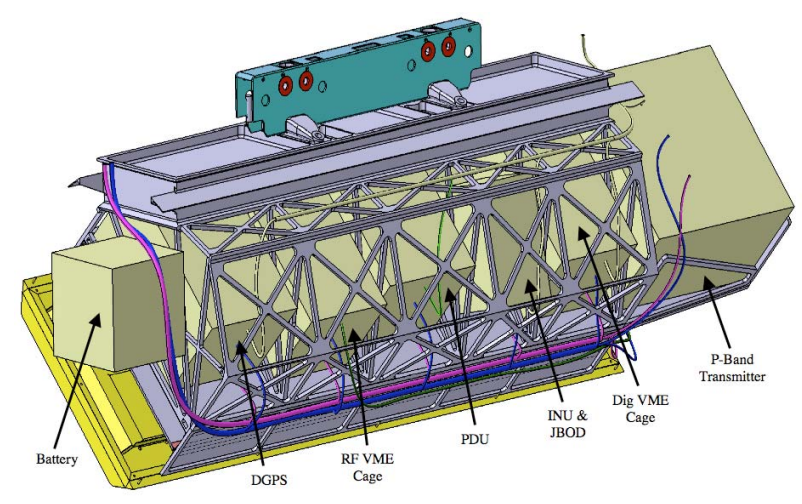

Fig. 4. Configuration of L-band radar electronics and antenna within the structure of the pod. Note the P-band transmitter is not part of the baseline system, but is shown to indicate that there is space in the pod for both the baseline L-band system and P-band as a second frequency.

variation of the array over temperature and time. Additionally, 
the antenna supports an elevation "monopulse" mode whereby the signals from the top and bottom half of the antenna can be recorded separately.

Figure 4 shows the configuration of the radar electronics embedded within the pod structure.

\section{Estimate of Power, Weight, Volume}

The estimated DC power for the L-band polarimetric RTI is just under $1.7 \mathrm{~kW}$ when the radar is transmitting. This is well within the capacity of the Gulfstream III or the Predator-B aircraft. The standby DC power should be on the order of 150 W. The active array antenna should weigh less than $40 \mathrm{~kg}$ since each T/R module weighs about $0.5 \mathrm{~kg}$. The remainder of the radar electronics in the payload bay should weigh less than $150 \mathrm{~kg}$ (approximately $8 \mathrm{~kg}$ for the RFES, $25 \mathrm{~kg}$ for the $\mathrm{DES}$, and $43 \mathrm{~kg}$ for cabling, power distribution, etc.).

Precise knowledge of motion and location is provided by the high precision INU and real-time differential GPS receivers [5]. Doppler centroid stability can be achieved by along track electronic beam-steering up to $\pm 20^{\circ}$ with a goal of $\pm 45^{\circ}$ linked to the INU attitude angle measurements. This dictates the radar design to utilize an active array antenna with transmit/receive (T/R) modules and phase shifters with a beam steering angle resolution of better than $1^{\circ}$.

Based on a data file provided by flight planning software, the UAVSAR will automatically initiate data takes at the appropriate locations throughout the flight. This approach was implemented on GeoSAR (a radar interferometric mapping system designed and built by JPL and currently operated by Earthdata International which is hosted on a Gulfstream II aircraft) with good results. Because of the autonomous requirement, this instrument must include BIT (Built In Test) capability and be able to determine failure at the unit level. A modular approach to delineation of logic functions in the instrument will assist in the addition of potential options in the future. Because the instrument is designed for modularity, reconfiguration for the addition of potential options or installation on a different platform should be feasible.

\section{Radar Modes}

The baseline mode for the single-antenna UAVSAR implementation is L-band Polarimetry (PolSAR). This mode combined with RTI will support not only deformation measurements (zero baseline case) but also high precision elevation mapping (non-zero spatial baseline) and polarimetric interferometry, e.g. for vegetation and volume scattering studies. The baseline system will also support Co-Polarized Monopulse (CoPM) measurements. This means that the signals recorded by the top and bottom halves of the antenna, can be recorded individually, which in essence provides for a very short baseline interferometric system. In this mode the system will not support a full quad-pol capability, only like (co-) polarized channels are recorded. Finally, the baseline system will support a Multi-Squint Differential IFSAR mode, allowing the azimuth beam to cycles through multiple aspect angles (e.g. fore, broadside, and aft) on consecutive pulse transmissions as shown in Fig 5. This capability will allow experiments to measure vector displacements, and to estimate vector displacements and atmosphere simultaneously.

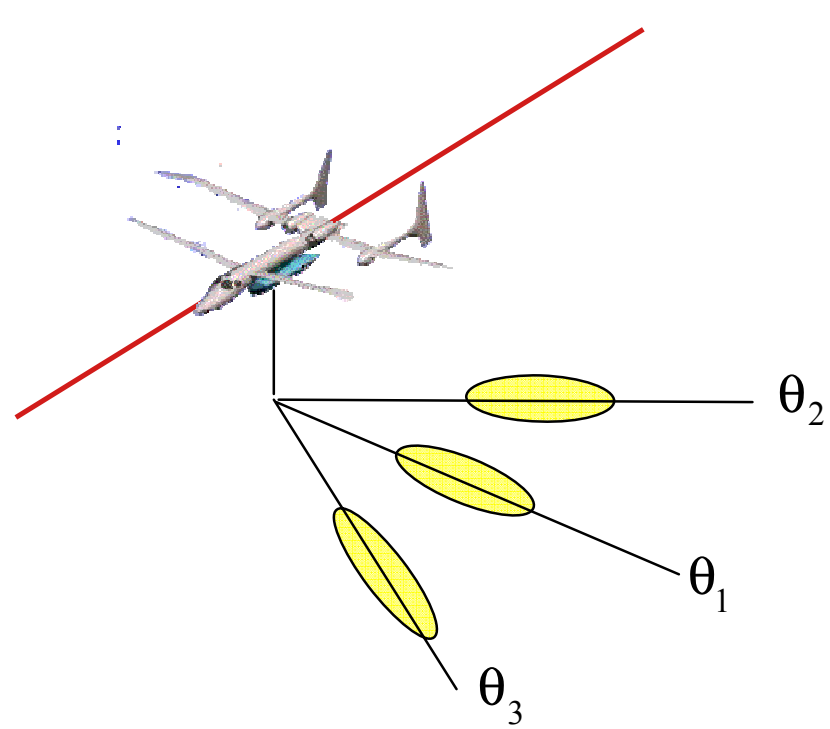

Fig. 5. Because the electronically scanned antenna has the capability to steer on a pulse-to-pulse basis, three simultaneous SAR images can be generated at three distinct and widely separated squint angles as illustrated above. This will allow the generation of vector deformation maps as well as offer the potential of solving for tropospheric distortions to the deformation signal.

The monopulse capability mentioned previously will be implemented to support modes for single or dual polarization acquisitions.

A second L-band polarimetric antenna is being built as part of the current project, with the intention of augmenting the system with a second L-band radar in the future. With two radars mounted on the aircraft, UAVSAR would support modes for single-pass across-track interferometric data acquisitions, including several polarimetric cross-track interferometry modes. Finally, if another antenna is mounted along the fuselage relative to the primary antenna, the system will also support a vector along track interferometry mode for either polarization.

\section{E. Science Measurement Error Budget}

The UAVSAR radar instrument is designed to be a precision interferometer to the extent it is possible, with careful attention to control and calibration of phase errors within the 
TABLE II

DEFORMATION ERROR SOURCES AND BUdGET

\begin{tabular}{|l|c|}
\hline Error Contribution Source & Error Contribution \\
\hline SNR Decorrelation & $0.2 \mathrm{~mm}$ \\
\hline Geometric/Temporal Decorrelation & $1.0 \mathrm{~mm}$ \\
\hline Flight Path Uncertainty* & $1.0 \mathrm{~mm}$ \\
\hline Instrument Phase & $0.6 \mathrm{~mm}$ \\
\hline Topography Uncertainty & $5.5 \mathrm{~mm}$ \\
\hline Atmospheric Distortion & $10 \mathrm{~mm}$ \\
\hline
\end{tabular}

antenna and electronics subsystems. There several interferometric error sources that are difficult to control for repeat pass systems however, and a certain degree of refinement of the interferometric parameters through the observations will be required, hence the ultimate performance of the system will be data dependent.

Table II introduces the major interferometric error components. The error is characterized in terms of deformation error, which is derived from phase errors in a single look ( $0.75 \mathrm{~m} \times 0.87 \mathrm{~m}$ pixels) arising from random and systematic effects in the interferometric observation, averaged to $20 \mathrm{~m}$ x $20 \mathrm{~m}$ resolution cells. The random phase effects are characterized through the interferometric correlation [4].

A radiated power of $1.7 \mathrm{~kW}$, required principally for good signal-to-noise ratio in cross-polarization channels, gives excellent co-polarization SNR, leading to very low thermal decorrelation and phase noise. With the expected very small baselines of less than $10 \mathrm{~m}$, geometric decorrelation is quite small. Temporal decorrelation can be arbitrarily high depending on the surface conditions (the extreme case is total decorrelation over water independent of repeat period), however here we assume that the surface decorrelation is at most 0.2. The actual amount of surface decorrelation varies depending on interval between observations and surface cover (e.g. vegetated, snow, urban, etc.). L-band has been shown to be very robust with regard to surface decorrelation for a large range of repeat intervals and surface covers. The "instrument phase" entry in the table refers to systematic two-way phase variability over the data take after calibration of the antenna steering states.

The absolute flight path can be reconstructed from the high precision differential GPS to an accuracy of a few $\mathrm{cm}$ at best. Thus the relative flight paths needed for interferometric processing can only be estimated to that level. This is insufficient for differential interferometry because errors in the relative flight path are indistinguishable from deformation errors, and introduce topographic errors as well. The error contribution noted in Table II due to flight path uncertainty of $1 \mathrm{~mm}$ assumes that relative flight paths can be refined from the data themselves, through estimation of residual offsets between processed images and subsequent modeling of residual motion from the offsets.

The two dominant error sources in the table are due to uncertainty in the topography and refractive variability of the atmosphere that introduces random phase distortions. The topographic term can be seen from the equation relating interferometric phase $\Delta \varphi$ to deformation $\Delta \rho_{d}$ and topographic height $z$ above a reference surface:

$$
\Delta \varphi=\frac{4 \pi}{\lambda} \Delta \rho_{d}-B \cos \left(\theta_{0}-\alpha\right) \frac{z}{\rho_{0} \sin \theta_{0}}
$$

where $B$ is the interferometric track separation (baseline) length, $\alpha$ is the track separation angle relative to a reference level, $\rho_{0}$ is the range of the reference surface for a given ground element, and $\theta_{0}$ is the look angle to the reference surface. For differential interferometry, we attempt to remove the topographic phase term with the use of a topographic map to derive the deformation $\Delta \rho_{d}$. Clearly for a non-zero baseline, any error in topography will incur a phase error that will be confused with deformation.

The best available global scale topographic data set is the Shuttle Radar Topography Mission (SRTM) data [6], and the known error characteristics of that product have been used to characterize the error due to topographic uncertainty. Improving knowledge in terrain height through improved

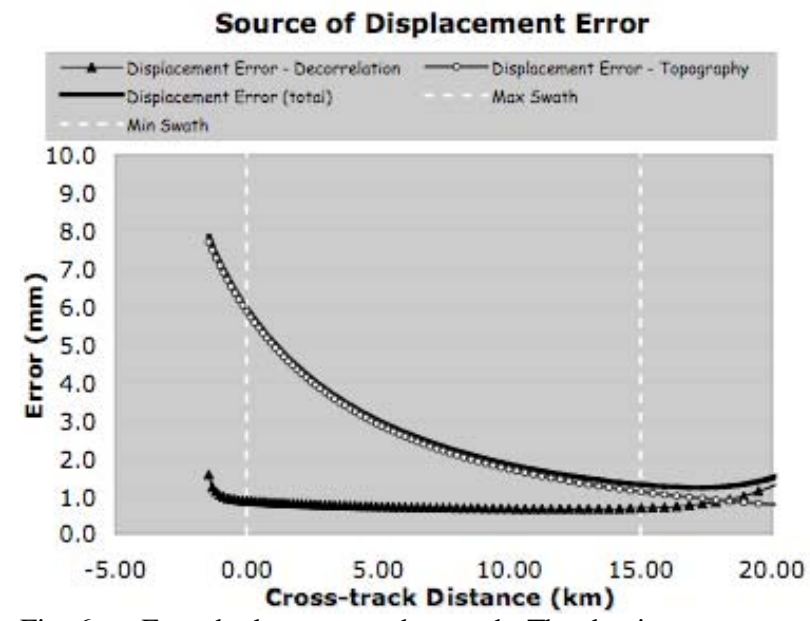

Fig. 6. Error budget across the swath. The dominant source of error in the near range is induced by error in knowledge of the topography (based on SRTM). By using higher accuracy topography products, from other sources or from repeat passes of UAVSAR with suitably long baseline, this error can be reduced.

topography maps or 3 pass techniques will improve this error. 


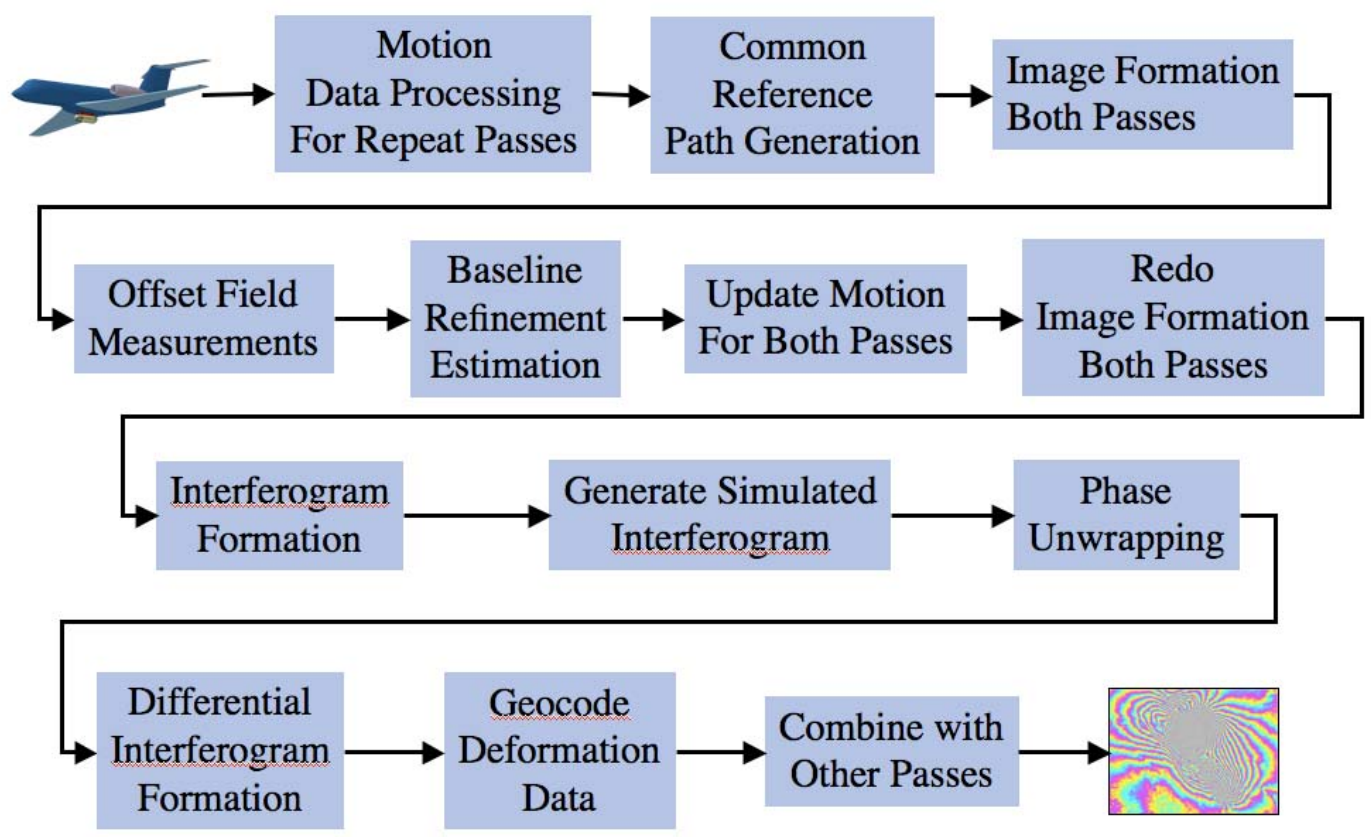

Fig. 7. Processing methodology for differential interfeometry for airborne RTI. Unlike processing of spaceborne RTI data, where the flight path is very smooth over time such that residual errors can be easily modeled after initial processing, airborne flight tracks are not known sufficiently well in advance. It is necessary to use the data themselves to refine the timevarying the baseline between flight tracks, reprocess the data with updated flight track information, then proceed with interferometric processing.

The atmospheric term has been studied at length [4,7,8], and is the only term that cannot be controlled through engineering design of the instrument or associated data sets. It can be mitigated however by collection of many repeated data sets, allowing segregation of the spatially and temporally random atmospheric term from a geophysical system that is varying slowly and somewhat predictably over time.

Fig. 6 shows how the errors (excluding atmospheric errors) behave across the radar swath.

\section{SCIENCE PROCESSING}

The processing flow for differential interferometry is shown in Fig. 7. The methods for differential inteferometric processing have been described at length in the literature (see [4] for a review), particularly for spaceborne data sets. In the standard approach, two images are formed from the best available flight track information, and an interferogram is formed. Using a digital elevation model and the best available interferometric baseline estimate, a simulated interferogram is formed, and the two interferograms are compared. Systematic differences in the phase are attributed to baseline errors, and a refined baseline is estimated. The refinement process usually consists of the estimation of a low order polynomial or fewparameter baseline model to affect the correction. Generally, then the phase of the simulated interferogram calculated with the refined baseline is subtracted from the data, leaving only the deformation phase, as represented in Eq. (2).

For airborne RTI, the principle is the same, in that a refinement of the baseline is needed, however the procedure must be modified and based on the data themselves. The baseline knowledge attainable with high precision differential GPS is insufficient to produce a smooth differential interferogram, so the refinement procedure allows for estimation of a baseline error that has the frequency content of the aircraft motion itself.

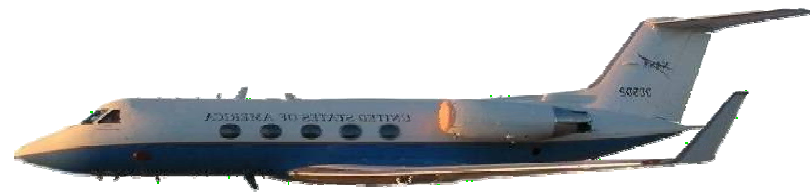

Fig. 8. The NASA Gulfstream III, the platform on which the UAVSAR pod-mounted radar will initially be deployed. As UAV platforms become available and flight-certified over science targetsof interest, the UAVSAR radar will be transitioned to a UAV-based system.

In order to have the needed spectral content and sensitivity to motion, the two-dimensional field of offsets of one image relative to the other is computed. The data are initially processed with motion-compensation to a common reference 
track. The range offset is proportional to the interferometric phase, and therefore carries the same information used in the conventional refinement approach. In addition, however, the along-track offset carries information about the local convergence or divergence of the flight paths, to subcentimeter accuracy. This information can be used to estimate the coefficients of a high-order Chebyshev polynomial model of the baseline error. Once the baseline is refined, because we choose a common reference track and the corrections to it are so large, the data must be reprocessed.

\section{DeVElopment Status}

The UAVSAR project has completed its critical design review and is currently in fabrication. The flight system will be ready for first flight tests in Fall 2006. The aircraft for which the radar pod will be initially configured will be the NASA Gulfstream III (Fig. 8). The Gulfstream is a research test-bed for NASA, the Air Force and other government agencies, a long-term asset for efficient experimentation at sub-sonic speeds (maximum $0.85 \mathrm{mach}$ ). It has a payload capacity of $1,180 \mathrm{~kg}$ with a full fuel load, and a range of 3,400 nautical miles when fully loaded with passengers (or equivalent payload). The pod will mount on the fuselage under the wings.

The pod will be portable to other piloted and unpiloted airframes, with some modification to the mounting hardware and housekeeping interfaces, but nominally with no change to the radar in the pod itself. We have shown that the UAVSAR radar pod can be accommodated on a Predator-B, a potential alternative platform that is a true UAV. The Predator-B has a payload capacity of $340 \mathrm{~kg}$, a power capability of $4.5 \mathrm{~kW}$, and a range of 5100 nautical miles.

This system will be the first civilian SAR to incorporate an electronically scanned array and is expected to provide a robust repeat pass interferometric mapping capability to the science community. The system has been designed with portability and extensibility as primary factors. With future upgrades, UAVSAR will be able to support the science community beyond solid earth, cryosphere, and land cover applications.

\section{ACKNOWLEDGEMENT}

This paper was written at the Jet Propulsion Laboratory, California Institute of Technology, under contract with the National Aeronautics and Space Administration. We would like to thank Total Aircraft Services who is modifying the GIII and supplying the pod for the pod interior figures and NASA Dryden for their expertise on aircraft systems and precision flying.

Reference herein to any specific commercial product, process, or service by trade name, trademark, manufacturer, or otherwise, does not constitute or imply its endorsement by the United States Government or the Jet Propulsion Laboratory, California Institute of Technology.

\section{REFERENCES}

[1] http://www.earth.nasa.gov/visions/stratplan/index.html.

[2] http://solidearth.jpl.nasa.gov/seswg.html

[3] http://solidearth.jpl.nasa.gov/gess.html

[4] Rosen, P. A., S. Hensley, F. Li, I. Joughin, S. Madsen, D. Goldstein (2000). Synthetic Aperture Radar Interferometry, Proc. IEEE, 88(3), 333-382

[5] Muellerschoen, R. and Bar-Sever, Y., "Aviation Applications of NASA's Global Differential GPS System", Proceedings of the 2nd AIAA "Unmanned Unlimited" Conf. and Workshop \& Exhibit, AIAA Paper 2003-6618, Sept. 2003.

[6] Hensley, S., R. Munjy, P. A. Rosen (2001). Interferometric Synthetic Aperture Radar, Digital Elevation Model Technologies and Applications: The DEM Users Manual, D. Maune, Ed., ASPRS, 142-206.

[7] Goldstein, R. M., Atmospheric limitations to repeat-track radar interferometry, Geophys. Res. Lett., vol. 22, 2517-2520, 1995.

[8] Hanssen, R.F. Radar Interferometry: Data Interpretation and Error Analysis. Kluwer Academic Publishers, Dordrecht, 2001. 